\title{
Genetic medicine gets real
}

\section{Gene therapists used to talk about permanently fixing 'broken' genes. But the emphasis has now shifted to treating conditions such as coronary disease and cancer using transient gene expression. Alison Abbott reports.}

T he language used by gene therapists has become noticeably more sober over the years. In the early 1990s, the field's pioneers raised hopes of curing single-gene disorders such as cystic fibrosis and severe combined immunodeficiency. Today, talk of cures has been replaced by guarded optimism about "encouraging" results. And the focus of attention has shifted away from genetic diseases towards using gene therapy as part of the therapeutic arsenal needed to confront major killers such as heart disease and cancer.

Two classical issues brought gene therapists down to Earth: efficacy and toxicity. The original idea of replacing missing or defective genes was compelling. But it soon became obvious that the vectors, such as weakened viruses, used to ferry the new genes into patients were highly inefficient. And in some instances, the vectors can also cause an adverse inflammatory reaction - as in the tragic case of the US teenager Jesse Gelsinger, whose death in September 1999 during a genetherapy trial cast a pall over the entire field. Even when genes are successfully and safely transferred, their activity typically tails off after only a short period.

Faced with these problems, many gene therapists have gone back to the laboratory bench, trying to solve these technical issues in animal models before returning to human trials. But others have kept one foot in the clinic, turning to diseases that could be approached with the imperfect tools available. "We started to think positive," says Ronald Crystal of the Cornell Medical College in New York. "What applications could we have for transient gene expression?"

\section{Growth potential}

The list of contenders is growing, with cancer and coronary-artery disease at the top. Attempts to treat single-gene diseases now account for only about an eighth of the worldwide roster of 500 or so approved clinical protocols. Trials using transient gene expression - in which a short period of activity by transferred genes is sufficient

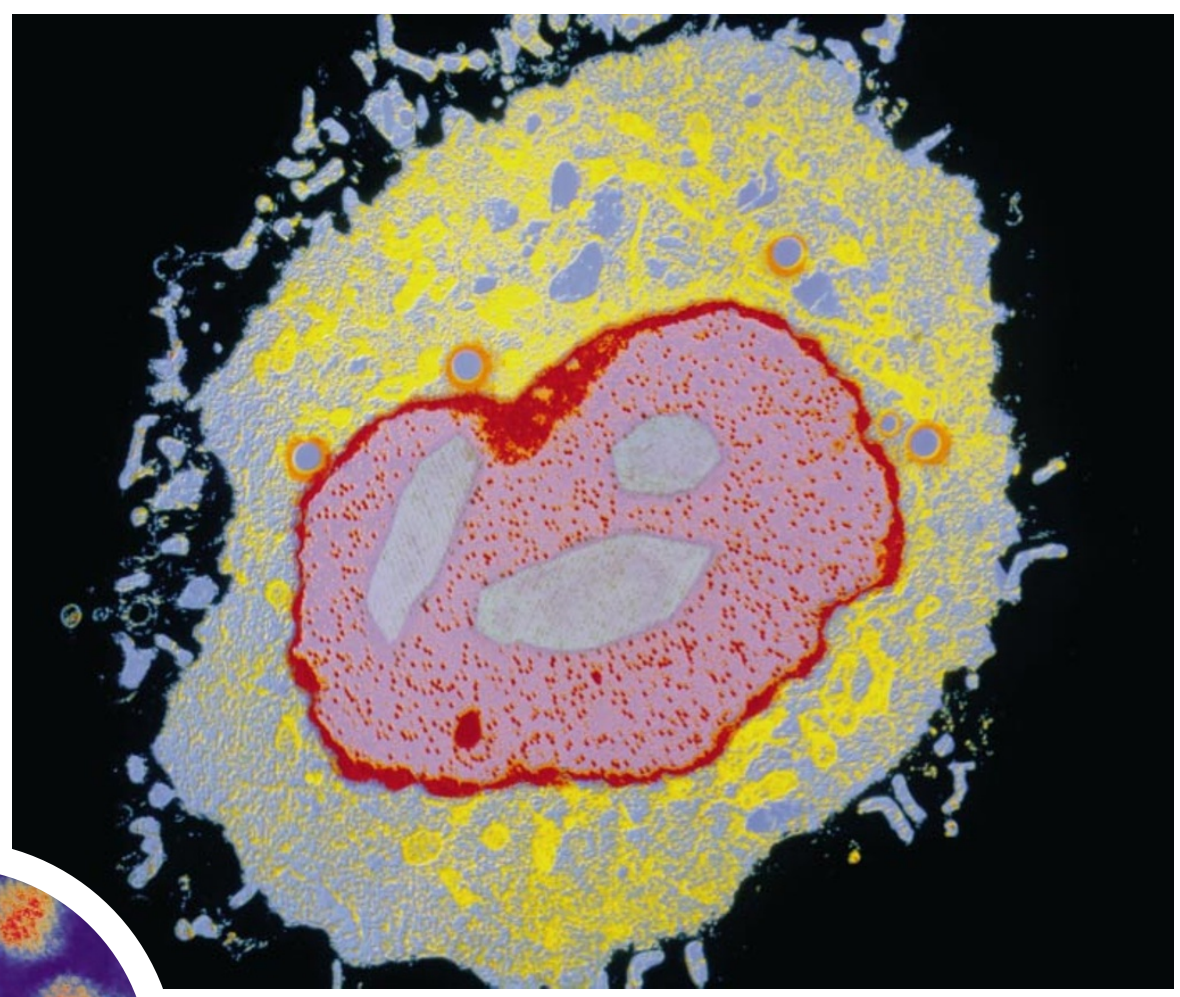

Gene ferries: adenoviruses (left), seen as red dots in the nucleus of this cultured human cell (above), can be used to introduce therapeutic genes into patients.

to treat a range of conditions - account for most of the rest.

Excitement over the potential of gene therapy to help treat coronary heart disease stems in part from work in Crystal's lab. In 1998, his team showed that introducing a gene encoding the protein vascular endothelial growth factor (VEGF) into the heart muscles of pigs caused new blood vessels to grow around blocked coronary arteries ${ }^{1}$.

Crystal introduces his therapeutic genes using adenoviruses - a major cause of common colds - modified so that they cannot cause disease. But because adenoviruses are such common infectious agents, our immune systems are primed to recognize them, so the vectors are rapidly destroyed. This undermines attempts at efficient and permanent gene transfer which are needed to treat conditions such as cystic fibrosis the focus of Crystal's earlier trials.

But the vectors' transience was no obstacle to Crystal's work with VEGF. "This is exactly the property we wanted for vascular disease," he says. "It's like flicking a master switch - once the blood vessels have been triggered to grow, we don't need the switch to be flicked again." Indeed, excessive growth of blood vessels is something that Crystal wants to avoid.

Crystal has since moved into the clinic, testing the technique in 31 patients undergoing coronary-bypass operations in a phase I clinical trial, designed to assess safety ${ }^{2}$. "Nothing about efficacy can be concluded with phase I trials," he stresses. Despite this, the patients reported some relief of symptoms, such as stress following exercise, and techniques for monitoring the growth of blood vessels indicated improvement in areas of heart muscle into which the vector had been injected.

Jeffery Isner of Tufts University in Boston is also working with VEGF. His phase I trial

\section{he expression of \\ transferred genes} need only be enough to kick-start a response. 
involved 85 patients with coronary heart disease and 110 with blockages in their peripheral circulation - a complication of conditions such as diabetes. Rather than using an adenoviral vector, Isner injects naked DNA encoding VEGF into muscle tissue in the vicinity of the blocked arteries. The efficiency of gene transfer in this method is very low, but Isner believes it will be sufficient to produce enough VEGF to trigger the growth of blood vessels.

Preliminary results from Isner's trial, for 13 patients with coronary-artery disease, were published last August ${ }^{3}$. Again, scanning techniques suggested that the treatment had promoted the growth of new blood vessels.

In the wake of Gelsinger's death - in an unrelated gene-therapy trial at the University of Pennsylvania - progress has slowed. Both Crystal and Isner are now conducting further safety trials to be sure that the small number of deaths among very sick patients in their phase I studies occurred because of the patients' underlying health problems, rather than as a result of the therapy.

\section{Stimulating work}

Meanwhile, scientists working in the commercial sector are gaining ground. In March, at a meeting in Orlando, Florida, of the American College of Cardiology, Berlex Biosciences of Richmond, California, reported positive results with fibroblast growth factor (FGF), which also promotes blood vessel growth. In this trial, adenovirus vectors containing the gene for FGF were injected into the coronary arteries of 60 patients with angina. A further 19 patients served as placebo controls, receiving injections that did not deliver FGF. The treated patients enjoyed greater relief from their symptoms. At the same meeting, Berlex reported similarly encouraging results from a placebo-controlled trial in peripheral vascular disease. The company is now gearing up to start phase III studies, which will use large enough numbers of patients to determine efficacy.

Given these encouraging developments, gene therapists are now thinking about other conditions that might be treated using growth-factor genes. Gene therapy involving VEGF could, for instance, help wounds to heal by promoting the regrowth of skin and blood vessels. Several groups are looking at the potential of platelet-derived growth factor for the same application. Crystal is also conducting experiments on rats to test whether the genes for VEGF and BMP7, one of a family of proteins that promote bone growth, could be used to fuse vertebrae after spinal surgery. Currently, orthopaedic sur-
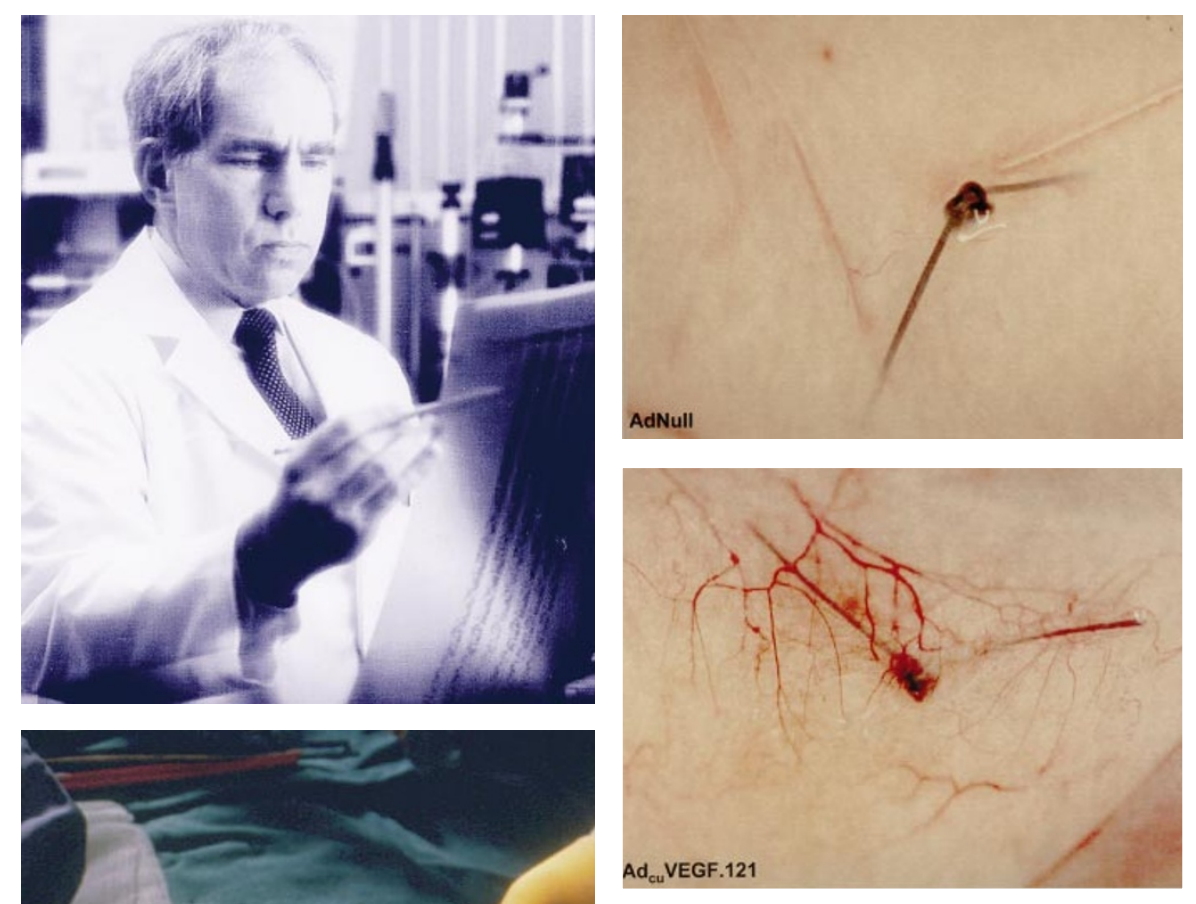
(1) 
$\mathrm{T}$ cells are activated by 'antigen-presenting cells', of which dendritic cells are the most important. Dendritic cells engulf and digest cellular debris, processing the resulting antigens so that they are carried on their surfaces. If the dendritic cells sense that these antigens represent a danger to the body, they also secrete 'co-stimulatory' molecules, and these activate the $\mathrm{T}$ cells that recognize the antigen involved. But triggering this sequence of events against antigens specific to tumour cells is a tough challenge.

One approach is to transfer a gene for a cytokine into tumour cells, and then to inject the resulting transgenic cells back into the patient. Cytokines are signalling proteins that help marshall immune responses, and previous research has shown that infusions of cytokines such as interleukin-2 (IL-2) can, by themselves, help shrink tumours. For example, in one trial of 255 patients with kidney cancer that had spread to secondary sites ${ }^{5}$, high-dose IL-2 caused remission in 12 patients, and a partial response in a further 24. But most patients experienced significant side-effects, particularly high fever.

Gene therapists are betting that using tumour cells to produce a transient burst of an appropriate cytokine will trigger dendritic cells more effectively, and so kick-start the chain of events to associate tumour antigens with danger — without causing generalized toxicity.

Early attempts at this strategy used tumour cells taken from the patients themselves. In 1998, for example, a team based at the Humboldt University in Berlin reported increased antitumour immune responses in 16 terminal melanoma patients vaccinated with their own cancerous cells transfected with genes for either interleukin-7 (ref. 6) or interleukin-12 (ref.7).

But most experts believe that tailoring therapy to individuals by genetically manipulating the patient's own cells is too timeconsuming to be a realistic clinical option. Instead, attention is now focusing on applying the same approach to cell lines maintained in culture that come from the same type of tumour as the patient's - which should share common antigens.

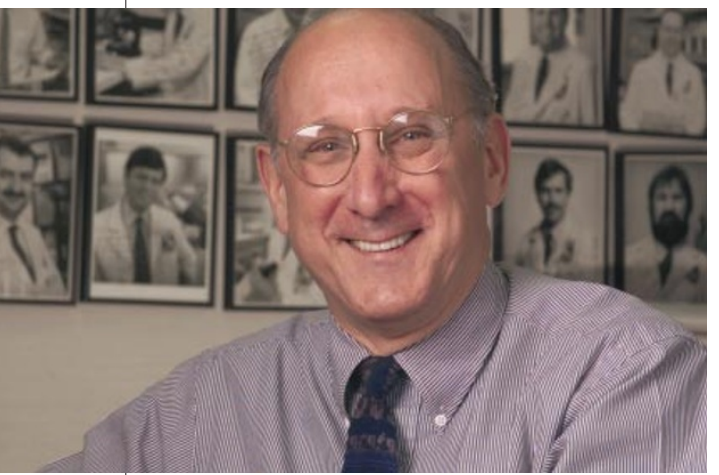

Positive approach: Steven Rosenberg is using transient gene therapy to tackle melanoma.
Early phase clinical trials are now being conducted by several companies, using the genes for cytokines including IL-2 and granulocyte-macrophage colony-stimulating factor. Prominent among these companies is Cell Genesys of Foster City, California, which is targeting melanoma plus kidney, prostate, pancreatic and lung cancers. Variations on the theme include transferring a second gene to the introduced cells

to promote the desired immune response even further. These include the gene for a protein that binds to a molecule called CD40, which promotes the maturation of dendritic cells, or for costimulatory molecules.

\section{In for the kill}

The disadvantage of 'off-theshelf' vaccines using cancer cell

lines is that some patients' tumours might carry a different set of antigens. In an attempt to avoid this problem, Vical, a company based in San Diego, is conducting phase I and II trials in which naked DNA encoding genes for cytokines or co-stimulatory proteins is injected into the patients' tumours. The idea is that the tumour cells will take up the DNA, and then transiently secrete proteins that will activate an immune response against the cells' antigens. The drawback, comments Pardoll, is that it is difficult to control the cells' uptake of DNA. Nonetheless, over the past two years Vical has reported at clinical research meetings encouraging results from patients suffering from melanoma, and prostate and kidney cancers.

An alternative approach to tackling tumours involves introducing the genes for cancer antigens inside viral vectors, in the hope that this will provide an appropriate context to associate the antigens with danger, and activate the immune system accordingly. Various early phase clinical trials are under way. Steven Rosenberg's team at the National Cancer Institute (NCI) in Bethesda, Maryland, for instance, has attempted to treat melanoma using adenoviruses carrying genes for specific antigens associated with the cancer ${ }^{8}$. Initial results were disappointing, however, with patients showing only weak immune responses.

In similar work, a team led by Jeffrey Schlom of the NCI and John Marshall from Georgetown University Medical Center in Washington DC, is tackling a range of cancers using a gene for a more generic antigen, called human carcinoembryonic antigen, spliced into the avipox virus ${ }^{9,10}$. Avipox is a member of the vaccinia virus family that infects birds but which cannot replicate in mammalian cells. The immune response against the tumour antigen appears to increase with each monthly injection, particularly if the

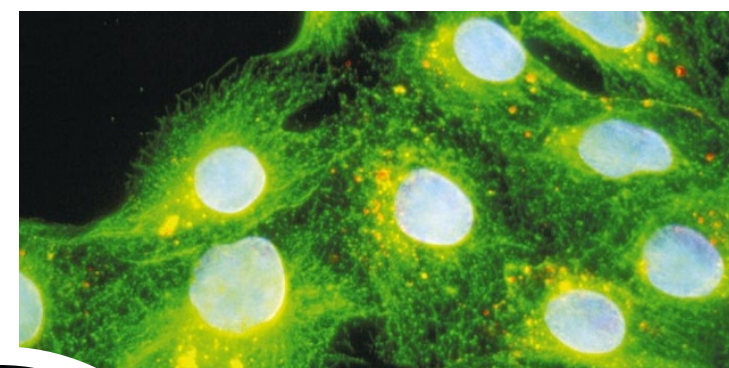
if

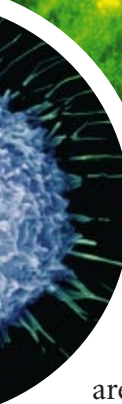

them with vaccinia.

At the 2001 meeting of the American Society of Clinical Oncology, held earlier this month in San Francisco, Schlom and Marshall's team announced the results of a small randomized trial of 18 patients with latestage colorectal cancer, whose survival time was increased against controls.

At this stage, researchers in the field are unsure which cancer vaccine strategies will emerge as the most promising. "It's far too early to know which way is best," says Rosenberg, who reviewed progress in tumour immunotherapy in last week's Nature". "We need to try lots of different approaches just to see which works, and we need to work creatively," he says. Researchers in the field also stress that cancer vaccines are not likely to be used in isolation, but alongside surgery, radiotherapy and chemotherapy.

Indeed, although gene therapy approaches to both vascular diseases and tumour immunotherapy are showing promise, they are still in their infancy. The true efficacy of these treatments will only be revealed by definitive phase III trials, involving thousands of patients. But with companies such as Berlex Biosciences now planning such trials, we shouldn't have to wait too long to find out whether transient gene therapy will deliver the goods.

Alison Abbott is Nature's senior European correspondent.

1. Mack, C. A. et al. J. Thorac. Cardiovasc. Surg. 115, 168-177

(1998).

2. Rosengart, T. K. et al. Circulation 100, 468-474 (1999).

3. Vale, P. R. et al. Circulation 102, 965-974 (2000).

4. Sato, N., Leopold, P. L. \& Crystal, R. G. J. Clin. Invest. 104, 855-864 (1999).

5. Fyfe, G. et al. J. Clin. Oncol. 13, 688-696 (1995).

6. Moller, P. et al. Br. J. Cancer 77, 1907-1916 (1998).

Sun, Y. et al. Gene Ther. 5, 481-490 (1998).

8. Rosenberg, S. A. et al. J. Natl Cancer Inst. 90, 1894-1900 (1998).

9. Marshall, J. L. et al. J. Clin. Oncol. 17, 332-337 (1999).

10. Marshall, J. L. et al. J. Clin. Oncol. 18, 3964-3973 (2000).

11. Rosenberg, S. A. Nature 411, 380-384 (2001). 